\title{
La «otredad» femenina: construcción cultural patriarcal y resistencias feministas
}

\section{Feminine «Otherness»: Patriarcal Cultural Construction and Feminist Resistance}

\section{RESUMEN}

Este ensayo tiene la finalidad de reflexionar sobre cómo el patriarcado ha construido una otredad femenina ubicada en un lugar de inferioridad, sobre todo a partir de la constitución de lo que llamaré el grupo nominador que marca y construye un signo que señala la inferioridad, en este caso anclado en la diferencia genital, cimiento de la arquitectura de los cuerpos sexuados, inventado al grupo signado como subalterno. A partir de la lectura crítica de producciones de los estudios culturales, y la utilización de sus categorías clave como la de cultura y la de identidad, me propongo visibilizar las resistencias a la construcción patriarcal de la otredad femenina, y a indagar acerca del potencial político que tiene la identificación como «mujeres» para la disputa cultural y el desmantelamiento de las trincheras del patriarcado.

Palabras clave: patriarcado, inferioridad femenina, resistencias, otredad.

\begin{abstract}
This essay aims to ponder over how patriarchy has built a female otherness located in a position of inferiority, especially since the creation of what I call the nominating group that builds brand and sign pointing inferiority -in this case anchored in the genital difference, which founded the architecture of sexed bodies, inventing the marked group as a subaltern group. From critical reading of productions of cultural studies, and the use of its key categories such as culture and identity, I intend to make visible the resistance to the patriarchal construction of female otherness, and inquire about the political potential It has identification as «women» for cultural dispute and the dismantling of the trenches of patriarchy.

Keywords: patriarchate, female inferiority, resistances, otherness.

\section{SUMARIO}

- Introducción. - La otredad construida. - Resistencias. - La disputa cultural. - Sujetas, identidades, identificaciones. - Reflexiones finales. - Bibliografía.
\end{abstract}

1 Abogada UNLP. Máster en derecho, UP. Máster en investigación aplicada en estudios feministas, de género y ciudadanía Universitat Jaume I. Máster en estudios interdisciplinares de género, USAL. Becaria Doctoral ICJUNLP. cano.julieta@gmail.com 


\section{Introducción}

Este ensayo tiene la finalidad de reflexionar sobre cómo el patriarcado ha construido una otredad femenina ubicada en un lugar de inferioridad, sobre todo a partir de la constitución de lo que llamaré el grupo nominador que marca y construye un signo que señala la inferioridad, en este caso anclado en la diferencia genital, cimiento de la arquitectura de los cuerpos sexuados, inventado al grupo signado como subalterno.

Para pensar en las categorías que recorren este ensayo, como lo son la de identidad, la de cultura y la de otredad, recurrí a una lectura selectiva de producciones de los estudios culturales, dado que es el campo disciplinar desde el que se ha trabajado más profundamente estos temas. El recurso a los estudios culturales lo entiendo pertinente porque desde el feminismo se plantea que la principal barrera para lograr la igualdad de género sigue siendo la cultural. A partir de la lectura crítica de algunos de los clásicos de esta disciplina (Williams, Hall), como también de autores y autoras contemporáneas y emergentes (Abu-Lughod), me propongo un ejercicio teóricoreflexivo para pensar en la otredad femenina, a partir de la utilización no sólo de las categorías de este campo, sino de cierta forma de construcción de sus razonamientos. El ejercicio consiste en tomar un conjunto de ideas, específicamente las dimensiones de la relación con el otro de Todorov y la idea de Hall para contar el patriarcado, para transformarlas en herramientas analíticas.

Me avocaré a repensar la categoría de cultura y de identidad como algo no-esencial; como también pensar sobre cómo la creencia de que las mujeres nos habíamos apropiado de esa construcción patriarcal de nuestra inferioridad es una estrategia del Patriarcado que tiende a invisibilizar las resistencias feministas que son muchas -aun cuando existen muchas mujeres que defienden el statu quo-. Por último, indagaré brevemente acerca del potencial político que tiene la identificación como «mujeres» para la disputa cultural y el desmantelamiento de las trincheras del patriarcado. La pregunta que recorre este trabajo es la siguiente: ¿cómo se constituyó un grupo privilegiado-grupo nominador-conformado por varones (como colectivo) que ostenta la capacidad de objetivar y someter a un grupo signado por ellos mismos como inferior: las mujeres (como colectivo)?

\section{La otredad construida}

El patriarcado, es definido por Heidi Hartmann como «un conjunto de relaciones sociales que tiene una base material y en el que hay unas relaciones jerárquicas y una solidaridad entre los hombres que les permiten dominar a las mujeres» (Hartmann, 1980: 97). Considero que dicha definición, a pesar de haber sido elaborada en la década de los ' 80 conserva vigencia por la forma en que la autora hace hincapié en esa «base material» del patriarcado, que se constituye por la apropiación de la fuerza de trabajo de las mujeres por parte del colectivo de varones. Dicha apropiación sucede mediante mecanismos de exclusión de las mujeres de determinados ámbitos (el ámbito de lo público: de lo político, del mercado) -con la consecuente reclusión de las mujeres en otros ámbitos (en ámbito de lo privado, de lo doméstico); y mediante la restricción de la sexualidad femenina (Hartmann, 1980). 
Existen re-definiciones más actuales de la categoría patriarcado, como por ejemplo la de Cristina Molina que lo define como «el poder de asignar espacios no sólo en su aspecto práctico colocando a las mujeres en lugares de sumisión, sino en su aspecto simbólico, es decir, nombrando y valorando esos espacios de las mujeres como "lo femenino"»(Molina, 2003: 124). Para esta autora, el patriarcado se construye como un topo-poder que opera como un todo-poder androcéntrico. Puedo pensar en que ambas definiciones son complementarias, que una operación es consecuencia de la otra. Lo cierto es que el feminismo académico ha profundizado sin descanso en la categoría patriarcado y lo ha matizado bastante; aunque sigo prefiriendo la conceptualización de Hartmann porque contiene los elementos que considero indispensables para la definición del patriarcado.

El patriarcado el fundamento de lo que Marcela Lagarde llama organización social patriarcal (en un intento de deshipostasiar el término). Esta organización social patriarcal apela a una noción de cultura de tipo estática, inmodificable en su esencia. El patriarcado se presenta en el imaginario popular como ahistórico, como resultado «natural» del contrato social ${ }^{2}$. El primer paso que me propongo es indagar acerca de cómo se construye la otredad en el marco de una organización social patriarcal y cómo dicha otredad es constitutiva de la subjetividad del grupo signado.

De acuerdo a Todorov (1998) en las relaciones de las personas con la otredad se encuentran presentes tres ejes: un primer eje axiológico, de juicio de valor que ubica al/ a la otro/a como igual o inferior a mí; un segundo eje praxeológico que determina el alejamiento o acercamiento en mi relación con el/la otro/a: «adopto los valores del otro, me identifico con él: o asimilo al otro a mí, o le impongo mi propia imagen; entre la sumisión al otro y la sumisión del otro hay un tercer punto que es la neutralidad o indiferencia» (Todorov, 1998: 195); y un tercer eje epistémico, en relación con mi conocimiento o ignorancia del / de la otro/a.

La conceptualización de Todorov me resulta útil para pensar la relación entre varones y mujeres en el marco de una organización social patriarcal. De este argumento se desprende la presencia de un grupo nominador que tiene el poder de nombrar y constituir a las-otras en un lugar de inferioridad. Simone de Beauvoir visibilizó en 1949 la construcción de la mujer como lo-otro, postulando que lo-uno es el varón, y esta visión no sería intercambiable: «por ejemplo, si para un pueblo los otros son los "extranjeros", para esos "extranjeros", los otros serán quienes les llaman así. Es decir, el sentimiento de los otros es recíproco. Con la mujer no ocurre eso. El hombre en ningún caso es el otro» (Varela, 2005: 84).

De Beauvoir señala que la construcción de lo-uno y lo-otro, en relación con varones y mujeres, es universal por estar presente en todas las culturas: el varón es

2 Este tipo de respuesta es aun más frecuente cuando se analizan en un sentido centro-periferia las conformaciones sociales de los países subalternos con culturas bien diferentes al modelo occidental, en donde el relativismo cultural juega un lugar central para justificar la no-crítica y la no-intervención de quienes se sitúan en una postura de corte progresista. Para indagar sobre críticas al contrato social, puede verse: Pateman, Carol (1995) El contrato sexual. México: Anthropos, UNAM. 
lo universal y la mujer lo particular: «la Humanidad es macho, y el hombre define a la mujer no en sí misma, sino con relación a él, no la considera como un ser autónomo [...] la mujer es lo inesencial frente a lo esencial. Él es el sujeto, él es lo absoluto; ella es lo Otro» (Beauvoir, 2013: 18)3. Esta aparente universalidad no sólo es un componente de la hegemonía (Wright, 1999) que ostenta la cultura patriarcal, sino también es uno de los argumentos para responder los cuestionamientos feministas impugnadores.

La apelación a una cultura inmodificable o esencial para impedir los cuestionamientos feministas (entre otros cuestionamientos) fue desarticulada -sin querer queriendo- por los estudios culturales que entienden que las identidades culturales son «dinámicas, fluidas, y construidas situacionalmente, en lugares y tiempos particulares» (Wright, 1999: 130-131). De aquí también se desprende la posibilidad de construcción, deconstrucción y reconstrucción identitaria de los/as sujetos/as que habitan las conformaciones culturales pensadas como dinámicas.

Pensar en la construcción uno-otra en función de los tres ejes planteados por Todorov, implica en primer término y en relación con el eje axiológico, que los varones se identifican como un grupo heterogéneo al interior pero homogéneo como colectivo, que se autoadjudica el poder de ubicar a las mujeres en un lugar de inferioridad. Esta construcción es palpable a lo largo de la historia: "para demostrar la inferioridad de la mujer, los antifeministas apelaron entonces no sólo a la religión, la filosofía y la teología, como antes, sino también a la ciencia: biología, psicología experimental, etc.» (Beauvoir, 2013: 25). Las producciones teóricas con la finalidad de ubicar a las mujeres como inferiores sin innumerables: desde Aristóteles hasta Rousseau, pasando por Hegel y Schopenhauer podemos encontrar una actividad política desmedida con el fin de marcar la frontera jerarquizada entre el grupo nominador y el grupo signado.

Pensar en el colectivo de varones como grupo nominador significa ubicar a los varones en un lugar de poder de nombrar, es decir aquel que tiene la posibilidad de construir a las mujeres como las-otras, aquel que tiene voz; en contraposición al grupo signado que es nombrado por los otros y no puede construir su propia realidad ni su propia historia. Si recorremos la historiografía occidental, las voces de mujeres en el siglo XVIII como Olympe de Gouges o Mary Wollstonecraft aparecen olvidadas y sólo recuperadas por los colectivos feministas, a la par que Jean Jaques Rousseau deviene una autoridad casi incuestionable de la filosofía política. El grupo nominador se erige en autoridad, en la voz autorizada para construir la historia, el grupo signado se convierte en víctima de esa construcción.

En un segundo momento praxeológico, en donde se marca la distancia entre lo-uno y la-otra, se adjudican valores y características a cada grupo de una manera jerárquica que posibilita la justificación de la sumisión. En este sentido

3 Esto ya lo planteaba la autora en 1949, año de la primera publicación de su obra. 
es útil cómo piensa Olsen (2009) la construcción binaria ${ }^{4}$ del mundo: la organización del mundo está dualizada y dichos dualismos, además de sexualizados, están jerarquizados. Esto significa que la comprensión del mundo en términos duales implica una (o varias) dicotomías: varón/mujer, razón/emoción, cultu$\mathrm{ra} /$ naturaleza, racional/irracional, objetivo/subjetivo, público/privado; y que lo masculino se ha situado en un lugar del par dual que ostenta una posición jerárquica respecto del otro, donde se ha ubicado a las mujeres, tradicionalmente visto como lo negativo, lo inferior. En este sentido, Virginia Woolf decía en 1929:

Durante todos estos siglos, las mujeres han sido espejos dotados del mágico y delicioso poder de reflejar una silueta del hombre de tamaño doble del natural. Sin este poder, la tierra sin duda seguiría siendo pantano y selva. Las glorias de todas nuestras guerras serían desconocidas. Todavía estaríamos grabando la silueta de ciervos en los restos de huesos de cordero y trocando pedernales por pieles de cordero o cualquier adorno sencillo que sedujera nuestro gusto poco sofisticado [...] Sea cual fuere su uso en las sociedades civilizadas, los espejos son imprescindibles para toda acción violenta o heroica. Por eso, tanto Napoleón como Mussolini insisten tan marcadamente en la inferioridad de las mujeres, ya que si ellas no fueran inferiores, ellos cesarían de agrandarse. Así queda en parte explicado que a menudo las mujeres sean imprescindibles a los hombres. Y también así se entiende mejor por qué a los hombres les intranquilizan tanto las críticas de las mujeres; por qué las mujeres no les pueden decir este libro es malo, este cuadro es flojo o lo que sea sin causar mucho más dolor y provocar mucha más cólera de los que causaría y provocaría un hombre que hiciera la misma crítica (Woolf, 2008: 28 -29) .

\section{Si defino a la hegemonía como proceso, como un:}

Complejo efectivo de experiencias, relaciones y actividades que tiene límites y presiones específicas y cambiantes. En la práctica, la hegemonía jamás puede ser individual [...] no se da de modo pasivo como una forma de dominación. Debe ser continuamente renovada, recreada, defendida y modificada. Asimismo, es continuamente resistida, limitada, alterada, desafiada por presiones que de ningún modo le son propias (Williams, 1997: 134).

4 Al respecto de la construcción dicotómica y binaria de la organización social, Judith Butler se pregunta: «¿Es la disolución de los binarios de género, por ejemplo, tan monstruosa o tan temible que por definición se afirme que es imposible, y heurísticamente quede descartada de cualquier intento por pensar el género?» (Butler, 2007:9). El propósito de la autora en su obra El género en disputa tiene que ver precisamente con poder repensarnos en un marco no-binario, en donde no tengamos necesariamente que encajar en alguna de las dos categorías -varón/mujer- que define el patriarcado, sino poder pensar en una organización social en donde no sea necesaria la adscripción genérica, y/o se pueda pensar en más de dos géneros. De hecho, la existencia de personas intersexuales ya pone en dudas que la categorización binaria sea exhaustiva, aunque aun hoy la organización social sigue teniendo en los hechos base binaria y jerarquizada.

5 Esto ya lo planteaba la autora en 1929, año de la primera publicación de su obra. 
La dimensión hegemónica del patriarcado deviene incuestionable. Y los movimientos feministas aparecen como protagonistas en el cuestionamiento de dicha hegemonía, pero siempre desde los márgenes. Podemos pensar por ejemplo el contenido peyorativo que tiene el término feminista: muchas mujeres que trabajan y militan por la igualdad inter-géneros, le huyen e intentan no identificarse con él por el estigma que pesa sobre el mismo. Puedo reconocer en ese estigma una construcción no inocente del patriarcado. Cuando indago acerca del significado del feminismo, son pocas las voces que lo identifican con un movimiento social y político que aboga por la igualdad inter-géneros; circula la idea de que el feminismo es un movimiento contra el varón, que postula la superioridad femenina y que pretende excluir al colectivo de varones de todos los lugares que tradicionalmente han ocupado. Es decir, muchas personas entienden feminismo como hembrismo, es decir, como machismo al revés. El patriarcado se ha encargado que las mujeres (y los varones) le huyan a la identificación como feministas y ha convertido el término en algo dudoso; y que ésto sea de esta manera no es casualidad, sino que una estrategia para desacreditar al movimiento al que de hecho las mujeres le debemos la emancipación.

En el marco de una organización social patriarcal, la superioridad masculina aparece como desideologizada ${ }^{6}$ y, como señala Wright (1999), se identifica esta construcción -resultado de las relaciones de poder inter-género- como si fuera el propio eje de la cultura misma, tanto en oriente como en occidente: «En su forma más segura, una ideología aparece como hegemónica. Esto es, se torna tan naturalizada, dada por hecho y 〈verdadera〉 que las alternativas están fuera de los límites de lo imaginable. [...] en su dimensión hegemónica, la cultura aparece como coherente, sistemática y consensuada» (Wright, 1999: 132). Por eso las acciones feministas son tan resistidas incluso por algunas mujeres: la igualdad inter-géneros se propone como tan disruptiva de la cultura, que su propuesta de una organización social alternativa todavía suele pensarse como imposible.

Volviendo a los ejes planteados por Todorov, en un tercer momento epistémico, se adjudican el saber completo de la-otra, aunque en realidad lo que existe en el caso en estudio, es una construcción ficticia, pero «científica» de ese saber, que los movimientos feministas van a cuestionar, ya incluso desde principios del siglo XX. Es interesante al respecto la observación que realiza Virginia Woolf cuando recorre el museo británico:

...la enorme cantidad de libros que había en el British Museum escritos por hombres sobre las mujeres. Sin duda tenía la culpa la campaña de las sufragistas. Debía de haber despertado en los hombres un extraordinario deseo de autoafirmación; debía de haberles empujado a hacer resaltar su propio sexo y sus características, en las que no se habrían molestado en pensar si no les hubieran desafiado (Woolf, 2008: 71).

6 Voy a entender ideología como «un sistema de significados, valores y creencias relativamente formal y articulado, de un tipo que puede ser abstraído como una "concepción universal"» (Williams, 1997:130). 
El grupo nominador tiene la capacidad de instalar como verdad aquello que inventa, supone, prefiere, le conviene, sobre el grupo signado. La construcción de la inferioridad es política y utiliza distintas herramientas, siendo la ciencia una de las vedette. Al respecto, son útiles las reflexiones Elias (1998) sobre la construcción de la alteridad ${ }^{7}$. El grupo autodesignado nominador de la otredad:

En todos esos casos el grupo más poderoso se ve a si mismo como gente «mejor», como dotado de una especie de carisma de grupo, como poseedor de un valor que comparten todos sus miembros mientras otros carecen de él. Es más, en todos los casos, la gente «superior» puede lograr que la gente menos poderosa se sienta como si le faltasen valores, es decir, como si fuese humanamente inferior (Elías, 1998: 82).

Pienso en cómo esta operación ha impactado en la construcción de la subjetividad femenina a lo largo de la historia, lo cual no hace otra cosa que alimentar la creencia de la superioridad del grupo nominador, tal como lo plantea Virginia Woolf con la metáfora del espejo ${ }^{8}$. El «espejo» del patriarcado está cimentado, entre otras cosas en la ciencia, la cual, equiparada a la verdad, nos definió históricamente como la falta, lo castrado -desde una perspectiva androcéntrica que se presenta como neutral.

Hay ciertas corrientes feministas que son criticadas - por el feminismo radical de los años '70 por ejemplo- por plantear una postura asimilacionista, que reconocería al varón como modelo, legitimando una organización social patriarcal androcéntrica, en donde no habría espacios para ser diferente, y en donde se tendrían derechos sólo en tanto y en cuanto nos acerquemos al modelo varón. Los distintos feminismos revelan que el antónimo de igualdad es desigualdad, y no diferencia. Se aboga por la construcción de un espacio en donde ser diferente no signifique ser inferior, y en donde se reivindique la diferencia como valiosa.

\section{Resistencias}

Muchas veces he escuchado decir que el derrocamiento del patriarcado en realidad beneficiaría tanto a varones y a mujeres, porque el patriarcado oprime a ambos

7 El trabajo de Elías es una reflexión sobre la construcción de la otredad en un vencindario obrero, en donde aparecen diferenciados dos grupos: el de los obreros que hace varias generaciones que habitan el barrio, y el de los «nuevos». En este trabajo al autor llama la atención sobre lo reduccionistas que pueden ser los análisis de la desigualdad social sólo centrados en el aspecto material. Además, señala la cohesión interna grupal como determinante, es decir lo que cohesiona es la pertenencia a un grupo que hacía dos o tres generaciones vivía en un lugar. La cohesión permite integración interna. Los/as otros/as aparecen como amenaza a cierto modo de vida: proteger la identidad de grupo, cerrar filas con los migrantes y asegurar su superioridad.

8 Resulta muy interesante para el análisis, recuperar la dimensión modélica que ostenta el grupo nominador. Para ello es muy clara una reflexión de Elías: «donde el diferencial de poder es grande, los grupos en posiciones marginadas por lo demás se suelen medir con la medida de sus opresores. Constatan que no cumplen las normas de aquéllos y se sienten ellos mismos inferiores [...] los marginados también experimentan su limitado poder emocionalmente como signo de su escaso valor, ante todo cuando las diferencias de poder son muy grandes y la subordinación resulta ineludible» (Resaltado propio, Elias, 1998:98-99). 
grupos. Siempre esa afirmación me hizo mucho ruido, dado que, aunque es verdad que los estereotipos de género impuestos son performativos de la subjetividad de varones y mujeres, y que esta organización social castiga severamente la disidencia, no podemos dejar de pensar en los privilegios que tienen los varones por haber nacido simplemente con el «signo que indica la superioridad». Tal como lo plantea Elías, los grupos que ostentan poder -grupo nominador- mantienen la cohesión interna gracias a un mecanismo de autoregulación grupal que implica la presencia de «la zanahoria y el garrote», lo que podría explicar falta de solidaridad inter-géneros:

Se mantiene a raya gracias a la gratificante participación de la superioridad humana del grupo y debido a la correspondiente elevación del amor propio y de la autoestima, reforzados por el continuo reconocimiento de la opinión grupal, y al mismo tiempo a través de las coacciones que se impone cada miembro a si mismo de acuerdo a los estándares y las normas del grupo (Elías, 1998: 123).

Para mantener la constitución del grupo nominador en un lugar de poder, es necesario que están presentes dos operaciones: exclusión y estigmatización (Elías, 1998) de las diferentes, de las marcadas. Al mismo tiempo, podríamos agregar que se intenta la construcción de una identidad esencial, tanto del grupo nominador como del grupo signado. Como bien señala Elías, no se trata de identificar condiciones individuales como fundamento de la exclusión y de la estigmatización, sino la mera pertenencia al grupo marcado por la diferencia genital-sexual como signo físico que marca la inferioridad, y un destino, es suficiente. La hegemonía patriarcal es la responsable de la falta de cohesión interna en el colectivo de mujeres, problema ya señalado por Simone de Beauvoir en El segundo sexo: al parecer las mujeres tenemos más solidaridad de clase con los varones que ofician de nuestros compañeros, parejas, maridos, etcétera, que con las propias mujeres con las que, aun inter-clase, compartimos la opresión específica del patriarcado sobre nosotras?.

9 Al respecto Butler se pregunta: «Si bien la afirmación de un patriarcado universal ha perdido credibilidad, la noción de un concepto generalmente compartido de las "mujeres", la conclusión de aquel marco, ha sido mucho más difícil de derribar. Desde luego, ha habido numerosos debates al respecto. ¿Comparten las "mujeres" algún elemento que sea anterior a su opresión, o bien las "mujeres" comparten un vínculo únicamente como resultado de su opresión? ¿Existe una especificidad en las culturas de las mujeres que no dependa de su subordinación por parte de las culturas masculinistas hegemónicas? ¿Están siempre contraindicadas la especificidad y la integridad de las prácticas culturales o lingüísticas de las mujeres y, por tanto, dentro de los límites de alguna formación cultural más dominante? ¿Hay una región de lo "específicamente femenino", que se distinga de lo masculino como tal y se acepte en su diferencia por una universalidad de las "mujeres" no marcada y, por consiguiente, supuesta? La oposición binaria masculino/femenino no sólo es el marco exclusivo en el que puede aceptarse esa especificidad, sino que de cualquier otra forma la "especificidad" de lo femenino, una vez más, se descontextualiza completamente y se aleja analítica y políticamente de la constitución de clase, raza, etnia y otros ejes de relaciones de poder que conforman la "identidad" y hacen que la noción concreta de identidad sea errónea» (Butler, 2007:50-51). A pesar de que comparto la idea de que la universalidad puede resultar esencialista con respecto al término mujeres, adhiero a una postura de identificación estratégica, pesar de los peligros que señala la autora y que abordaré más adelante en este trabajo. 
El grupo subalterno suele verse como amenaza para el grupo nominador, en términos de Elías, aunque en el caso de las mujeres, éstas no aparecerían como amenaza a cierto modo de vida de los varones per se, sino que su emancipación sí se convertiría en amenaza; dado que la superioridad masculina usufructúa los trabajos y productos del trabajo de las mujeres, porque el patriarcado según Hartmann (1980) construye su base material en las expropiaciones que realiza a las mujeres a través a la división sexual del trabajo, y gracias al efecto espejo señalado por Virginia Woolf.

Tal es así, que las disidencias dentro del grupo nominador son castigadas severamente. Quien ose cuestionar dentro del grupo las normas que lo constituyen, es echado fuera del mismo, y feminizado. Pensemos en lo que sucede con los varones disidentes del modelo heterosexista instaurado por el patriarcado.

\section{La disputa cultural}

Para pensar en la disputa cultural, me resulta útil el análisis de Hall (2010) cuando piensa en cómo se cuenta la cultura nacional, para pensar en cómo se cuenta el patriarcado. Hall señala cinco elementos principales: la narrativa [patriarcal], lo que incluiría la historia, los medios y la cultura popular. El segundo elemento lo constituyen los orígenes, la continuidad, la tradición, y la eternidad. El tercer y cuarto elemento serían la invención de la tradición y el mito fundacional. El último elemento es la noción de pueblo.

Los primeros cuatro elementos pueden resultar útiles para explicar una organización social patriarcal con fuerte anclaje en una cultura machista. Por ejemplo, repasando el mito fundacional: el mensaje de dominación de Eva por Adan se reproduce a lo largo de todas las culturas pre cristianas y no cristianas:

En épocas remotas, las mujeres se sentaban en la proa de la canoa y los hombres en la popa. Eran las mujeres quienes cazaban y pescaban. Ellas salían de las aldeas y volvían cuando podían o querían. Los hombres montaban las chozas, preparaban la comida, mantenían encendidas las fogatas contra el frío, cuidaban a los hijos y curtían las pieles de abrigo. Así era la vida entre los indios onas y los yaganes, en la Tierra del Fuego, hasta que un día los hombres mataron a todas las mujeres y se pusieron las máscaras que las mujeres habían inventado para darles terror. Solamente las niñas recién nacidas se salvaron del exterminio. Mientras ellas crecían, los asesinos les decían y les repetían que servir a los hombres era su destino. Ellas lo creyeron. También lo creyeron sus hijas y las hijas de sus hijas (Galeano, 2013: 41-42).

Me interesa pensar brevemente en los textos culturales como componentes imprescindibles de la producción y reproducción de la cultura patriarcal, y en el rol que desempeña la tradición ${ }^{10}$. Ésta es un elemento vital para mantener la he-

10 Pienso en la tradición de la manera que utiliza la categoría Hall en su texto, es decir como una construcción que honra el pasado (también construido) y recupera la experiencia de generaciones para perpetuarla. 
gemonía patriarcal, sobre todo retomando el concepto de Williams de «tradición selectiva» como «una versión intencionalmente selectiva de un pasado configurativo y de un presente preconfigurado, que resulta entonces poderosamente operativo dentro del proceso de definición e identificación cultural y social» (Williams, 1997: 137). La cuestión está en quiénes son los que eligen las tradiciones, y aquí otra vez estoy hablando de las potestades de un grupo nominador: «[...] a un nivel más profundo, el sentido hegemónico de la tradición es siempre el más activo: un proceso deliberadamente selectivo y conectivo que ofrece una ratificación cultural e histórica de un orden contemporáneo» (Williams, 1997: 138).

La dimensión cultural incluye a la tradición. Hay consenso en que las luchas feministas intentan provocar un cambio cultural, en donde es necesario destruir las trincheras en donde se legitima el patriarcado. Esta disputa cultural puede ir desde la producción de una telenovela inspirada en los principios feministas de organización social -como la que da cuenta Abu-Lughod (1995)- hasta la sanción de leyes y la modificación de los planes de estudios en todos los niveles educativos, entre muchas otras acciones.

Sin embargo, incluso las mejores intenciones pueden verse obstaculizadas por la naturalidad con que se asume el paradigma patriarcal. Aun guionando una telenovela mainstream con parlamentos feministas, cada mujer resignificará aquello que ve por la televisión de acuerdo a su propia cotidianeidad ${ }^{11}$. Tal como lo plantea De Certau para otro texto cultural como es el libro, éste es una construcción del/ de la lector/a:

...aparece entre el texto y sus lectores la reciprocidad que ocultaba, como si, al retirarse aquélla, se dejara ver la pluralidad indefinida de las «escrituras» producidas por unas lecturas. La creatividad del lector crece a medida que decrece la institución que la controlaba [...] Hoy, son los dispositivos sociopolíticos de la escuela, de la prensa o de la TV los que aíslan de sus lectores el texto poseído por el maestro o por el productor. Pero detrás del decorado teatral de esta nueva ortodoxia, se oculta (como ayer ya era el caso) la actividad silenciosa, transgresora, irónica o poética, de lectores (o televidentes) que conservan su actitud de reserva en privado y sin que 1o sepan los «maestros» (De Certeau, 2000: 185).

De todas maneras, el resultado de una acción de este tipo, siempre es imprevisto: se pueden resignificar cuestiones no pensadas como en el caso de Mothers in the

11 Al respecto resulta ilustrativo el análisis de Abu-Lughod: «La televisión vuelve obvio el hecho de que los mismos textos culturales tienen importancias diferentes en contextos distintos. Cuando Zaynab interpreta una escena como la del casamiento de una mujer de sesenta años como una diferencia cultural -vinculada a la región, al modo de vida ya la moral-, se debe a que tiene tantas desventajas en términos de clase y de educación que no logra comprender las intenciones de la más privilegiada creadora del programa. Para Al-Assal -que trabaja como política opositora dentro del contexto nacional de un país poscolonial y que discute con colegas intelectuales, críticos y políticos en El Cairo y a lo largo del mundo árabe mientras trata de reformar al público-, este episodio pretendía representar una opción feminista revolucionaria y esclarecedora. Sólo una etnografía móvil puede hacer justicia a los modos en los que se intersectan esos mundos diferentes. Y esta intersección debe formar parte de toda descripción densa de la televisión.» (Abu-Lughod, 2005:71-72). 
House of Love (Abu-Lughod, 1995) o puede ser una puerta para acciones emancipadoras y reivindicativas. De hecho, la producción de textos culturales que intenten subvertir el orden pueden ser una de las causas de las emergencias de nuevas sujetas: empoderadas, subversivas, disidentes, es decir, aquellas que no caben en la categoría «mujer» tal como la pensó y diseñó el patriarcado.

\section{Sujetas, identidades, identificaciones}

En la línea de la no-esencialidad de la cultura, hay que retomar el debate del feminismo postestructuralista sobre el sujeto «mujeres». ¿Existe un sujeto mujeres? Actualmente nos encontramos transitando un momento de crisis identitaria de los/as individuos debido al descentramiento del sujeto (Hall, 2010), momento en que se cuestiona la identidad de tipo esencial creada por la Ilustración: «El sujeto, previamente experimentado como poseedor de una identidad estable y unificada, se está volviendo fragmentado; compuesto, no de una sola, sino de varias identidades, a veces contradictorias y sin resolver» (Hall, 2010: 365).

La emergencia de una posmodernidad que cuestiona una identidad única de los sujetos, y que piensan a la identidad como una construcción socio histórica tiene como dos vertientes: por un lado cuestiona la existencia del sujeto «mujeres», pero por otro permitiría pensar la emergencia de nuevos sujetos con identidades no esenciales ni estables, pero articulables en la lucha contra la opresión. En esta línea, Butler plantea que, aunque:

...la teoría feminista ha asumido que existe cierta identidad, entendida mediante la categoría de las mujeres, que no sólo introduce los intereses y los objetivos feministas dentro del discurso, sino que se convierte en el sujeto para el cual se procura la representación política (Butler, 2007: 45-46).

La autora señala los peligros de este tipo de construcción arguyendo que «[1]a movilización de las categorías de identidad con vistas a la politización siempre está amenazada por la posibilidad de que la identidad se transforme en un instrumento del poder al que nos oponemos» (Butler, 2007: 32). La construcción de un sujeto implica necesariamente exclusiones, un antagonista a quien oponerse, pero las exclusiones son naturalizadas e inmediatamente después, invisibilizadas. Pensar que «mujeres» indicaría una identidad común que engloba a todas las personas de sexo femenino no sólo puede resultar esencialista, sino también homogeneizador de un grupo de personas que están atravesadas por otras categorías, que resulta en una opresión diferenciada, en donde las diferencias se pierden en perjuicio de «las más diferentes».

Volviendo a Hall (2010), el autor señala la importancia que tuvo el movimiento feminista para el descentramiento del sujeto, por la denuncia de la división público/privado y la consigna de «lo personal es político» (pensemos en los dualismos de Olsen); por traer a la arena pública cuestiones tradicionalmente consideradas del ámbito privado: la familia, la sexualidad, el trabajo doméstico, la división sexual del trabajo, la violencia, etcétera. Además «politizó la subjetividad, la identi- 
dad y los procesos de identificación (como hombres / mujeres, madres/ padres, hijos/hijas)» (Hall, 2010: 379). En este contexto, y aún a pesar de los "peligros» señalados por Butler, soy de la opinión de que dentro de la configuración social actual, la identificación como sujeta política mujer es relevante porque permite articular las luchas contra el patriarcado, «reclamar acceso a "recursos y garantías de derechos"» (Segato, 2007: 22) e incluso posicionarnos en un lugar de impugnación. Es lo que Segato identifica como «...el esencialismo autorizado o "estratégico", propuesto por una autora cuyo potencial crítico está por encima de cualquier duda corno Gayatri Spivak (1987)» (Segato, 2007: 5) ${ }^{12}$.

La identidad es posible de ser transformada y pensada históricamente en diálogo con los sistemas culturales que habitamos, convirtiéndose entonces en una «fiesta móvil» (Hall, 2010). Es pertinente pensar en cómo estamos constituidos/as: no soy solo mujer, soy blanca, soy heterosexual, soy clase media, soy universitaria, soy todo eso, soy distintos momentos y distintas temporalidades habitando un cuerpo. Analizar la interseccionalidad deviene necesario en tiempos de identidades migrantes y constitutivas de «fiestas móviles». Hall visibiliza el hecho de que: «si sentimos que tenemos una identidad unificada desde el nacimiento hasta la muerte, es sólo porque construimos una historia reconfortante o "narrativa del yo" sobre nosotros mismos (Hall 1990). La identidad totalmente unificada, completa, segura y coherente es una fantasía» (Hall, 2010: 365).

Si el primer paso es reconocer quien creo que soy hoy y ahora, paso siguiente propuesto por los diversos feminismos es reflexionar acerca de si esas categorías son representativas de mi misma: si la categoría «mujer» implica, en el marco de una organización social patriarcal que debo ser sumisa y estar al servicio de las necesidades masculinas, quizá impugne el término y elija definirme diferente o no definirme. Esto, como se apuntó, posibilita la emergencia de sujetos/as transformados/as o incluso de nuevos/as sujetos/as mucho más allá de lo que podríamos prever cuando hacemos el guion de una telenovela feminista. Pero también puede ser que me apropie del término «mujer» y lo resignifique, instalando un significado diferente, con potencial político subversivo que cuestione el orden profundamente, tal como lo proponen ciertos feminismos.

12 En contraposición a esta idea, Butler: «En realidad, la división en el seno del feminismo y la oposición paradójica a él por parte de las "mujeres" a quienes dice representar muestran los límites necesarios de las políticas de identidad. La noción de que el feminismo puede encontrar una representación más extensa de un sujeto que el mismo feminismo construye tiene como consecuencia irónica que los objetivos feministas podrían frustrarse si no tienen en cuenta los poderes constitutivos de lo que afirman representar. Este problema se agrava si se recurre a la categoría de la mujer sólo con finalidad "estratégica", porque las estrategias siempre tienen significados que sobrepasan los objetivos para los que fueron creadas. En este caso, la exclusión en sí puede definirse como un significado no intencional pero con consecuencias, pues cuando se amolda a la exigencia de la política de representación de que el feminismo plantee un sujeto estable, ese feminismo se arriesga a que se lo acuse de tergiversaciones inexcusables». (Butler, 2007:51). 


\section{Reflexiones finales}

En este breve ensayo, intenté pensar acerca de cómo el patriarcado nos construyó subalternas, como grupo signado, sobre todo a partir de los aportes de Todorov y de feministas ineludibles como Simone de Beauvoir y Virginia Woolf.

Así como se presentaron las dinámicas relacionales entre el grupo nominador y el grupo signado y los procesos de subjetivación femenina diseñados por el patriarcado, también se remarcaron las resistencias y la capacidad de agencia de las mujeres para la subversión del orden de género: es la capacidad de reflexividad de la agente lo que le permite el cambio social, en un aluvión de actividades que tienden a reproducir la estructura patriarcal en la cual se enmarcan. Si entendemos a la acción que «nace de la aptitud del individuo para "producir una diferencia" en un estado de cosas o curso de sucesos preexistentes» (Giddens, 2011: 51), esto nos permite explicar la centralidad que adquiere la agente en la reproducción de las prácticas sociales, y en la creación de instituciones; es decir, en la producción y reproducción de las estructuras sociales, pero también en la posibilidad del cambio social.

Por último, y en la misma línea, se presenta para el debate el potencial político que implica identificarse como mujeres para resignificar ese término, -a pesar de los riesgos que se corren al asumir esta posición reseñados por Judith Butler y anclados en el peligro de la reificación identitaria- y, desde allí, impugnar esta organización social en donde somos nombradas y construidas en un lugar de inferioridad. Poder pensar en la posibilidad del cambio en pos de una organización social feminista, es un triunfo de los movimientos feministas que de-construyen de a poco la hegemonía patriarcal, y así la desnaturalizan, dejando de pensar en que es la única forma de organización posible.

\section{BIBLIOGRAFÍA}

Aвu-Lughod, Lila (2005): «La interpretación de las culturas después de la televisión», Etnografías contemporáneas, N $\mathrm{N}^{\mathrm{0}} 1$, pp. 57-89.

Beauvorr, Simone de (1949): El segundo sexo, Buenos Aires, Debolsillo, 2013.

ButLer, Judith (1999): El género en disputa. El feminismo y la subversión de la identidad, España, Paidós, 2007.

De CERTEAU, Michel (1979): La invención de lo cotidiano, México, ITESO, 2000.

ELÍAs, Norbert (1976): «Ensayo teórico sobre las relaciones entre establecidos y marginados», En: Weiler, Vera (Comp.) La civilización de los padres y otros ensayos, Bogotá, Norma, 1998.

Galeano, Eduardo (1982): Memorias del fuego I, Buenos Aires, Siglo XXI Editores, 2013.

GIDDENS, Anthony (1995): La constitución de la sociedad: bases para la teoría de la estructuración, Buenos Aires, Amorrortu, 2011.

HALl, Stuart (1992): «La cuestión de la identidad cultural», En: Restrepo, Eduardo; Walsh, Catherine y Vich, Víctor (eds.) Sin Garantías. Trayectorias y problemáticas en estudios culturales, Ecuador, Envión Editores, 2010. 
HARTMAnN, Heidi (1980): «Un matrimonio mal avenido, hacia una unión más progresiva entre feminismo y marxismo», Zona Abierta, 2, pp. 85-113.

Molina, Cristina (2003): «Género y poder desde sus metáforas. Apuntes para una topografía del patriarcado», En: Silvia Turbert (ed.) Del sexo al género. Los equívocos de un concepto, Valencia, Cátedra- Universitat de València, pp. 123-159.

OlsEN, Frances (1990): «El sexo del derecho», En: Ávila Santamaría, Ramiro, Salgado, Judith y Valladares, Lola (Comps.) El género en el derecho. Ensayos críticos, Ecuador, Ministerio de Justicia y Derechos Humanos, 2009.

SEgato, Rita (2007): La Nación y sus otros. Raza, etnicidad y diversidad religiosa en tiempos de Política de la Identidad, Buenos Aires, Prometeo.

Todorov, Tzvetan (1998): La conquista de América. El problema del otro, Madrid, Siglo XXI.

VARELA, Nuria (2005): Feminismo para principiantes, Barcelona, Ediciones B.

Williams, Raymond (1977): Marxismo y Literatura, Buenos Aires, Manantial, 1997.

Woolf, Virginia, (1929): Una habitación propia, Barcelona, Editorial Seix Barral, 2008 (traducción al español por Laura Pujol).

Wright, Susan (1999): «La politización de la cultura» En: Boivin, Mauricio, Rosato, Ana y Arribas, Victoria Constructores de Otredad, Buenos Aires, Eudeba.

Recibido el 4 de enero de 2016

Aceptado el 16 de julio de 2016 BIBLID [1132-8231 (2016): 49-62] 